\title{
Efficacy of a 24-Week Aerobic Exercise Program for Osteopenic Postmenopausal Women
}

\author{
M. Y. Chien, ${ }^{1}$ Y. T. Wu, ${ }^{1}$ A. T. Hsu, ${ }^{2}$ R. S. Yang, ${ }^{3}$ J. S. Lai $^{4}$ \\ 'School and Graduate Institute of Physical Therapy, Medical College, National Taiwan University, Taipei, Taiwan, ROC \\ ${ }^{2}$ Department of Physical Therapy, Medical College, National Chen-Kung University, Tainan, Taiwan, ROC \\ ${ }^{3}$ Department of Orthopedics, Medical College, National Taiwan University, Taipei, Taiwan, ROC \\ ${ }^{4}$ Department of Physical Medicine and Rehabilitation, Medical College, National Taiwan University, Taipei, Taiwan, ROC
}

Received: 11 October 1999 / Accepted: 11 August 2000 / Online publication: 22 December 2000

\begin{abstract}
Osteoporosis is one of the most common skeletal disorders affecting postmenopausal women. The purpose of this study was to investigate whether a 24 -week program of aerobic high-impact loading exercise was beneficial for enhancing physical fitness and bone mineral density (BMD) in osteopenic postmenopausal women. Forty-three postmenopausal women aged 48-65 years participated in this study. The BMD of the spine (L2-L4) and right femoral neck of each woman was below 1 SD of the mean of premenopausal women, as examined by dual X-ray absorptiometry. The assignment of subjects into exercise or control group was not randomized but based on each subject's anticipated compliance to the 6-month long exercise program. Twentytwo subjects joined the exercise group and attended the training programs and 21 served as the control group. Exercise programs included treadmill walking at an intensity above $70 \%$ of maximal oxygen consumption $\left(\mathrm{VO}_{2} \max \right)$ for 30 minutes, followed by 10 minutes of stepping exercise using a 20 -cm-high bench. The program was conducted three times per week for 24 weeks. Physical fitness measurements included testing of flexibility, muscular strength and endurance, body composition, and cardiopulmonary fitness. The results showed that the quadriceps strength, muscular endurance, and $\mathrm{VO}_{2} \mathrm{max}$ in the exercise group had significant improvements, whereas no improvement was found in any of the physical fitness parameters in the control group. The BMD of the L2-L4 and the femoral neck in the exercise group increased $2.0 \%(P>0.05)$ and $6.8 \%(P<$ $0.05)$ and those in the control group decreased $2.3 \%(P<$ $0.05)$ and $1.5 \%(P>0.05)$, respectively. In conclusion, aerobics combined with high-impact exercise at a moderate intensity was effective in offsetting the decline in BMD in osteopenic postmenopausal women.
\end{abstract}

Key words: Aerobic exercise - Physical fitness - Osteopenia - Postmenopausal women

Osteoporosis is recognized as a major public health problem with significant medical, social, and economic consequences [1-4]. The incidence of osteoporotic fractures has been increasing and half of the elderly female population in most western as well as Asian countries are affected [5-9].

Correspondence to: $\mathrm{Y} . \mathrm{T}$. Wu
Menopause was considered as the major cause of postmenopausal osteoporosis. Decreasing mechanical stimulation with increasing age was an important confounding factor $[10,11]$. Exercise was reported to play an important role in maintaining or increasing bone density $[2,5,11]$. With exercise training, involutional bone loss might be reversed without the untoward side effects of medical therapy and could result in improvement of physical fitness and quality of life [12-14].

Some of the related studies, especially those with crosssectional design, have demonstrated a significant relationship between physical activity and bone mass [15-17]. Physically active subjects have significantly higher BMD than their age-matched sedentary controls [18, 19]. Results from longitudinal studies, however, provide conflicting conclusions, partly because of the difficulties in conducting and controlling compliance in long-term exercise programs. Other problems included the lack of adequate controls [20], poor control of confounding factors [21], and the choice of measurement sites [22].

Several studies have shown that exercise of relatively short duration did not enhance BMD, but declines in BMD were reported in non-exercising groups [23-25]. Several well-controlled studies supported a positive effect of exercise on BMD, indicating either less reduction or more gain in BMD for the training group compared with the control group [26-28]. The "optimal exercise regimen," however, has not been identified. Previous studies have used general weight-bearing activities as the main form of exercise intervention. More recent studies have suggested that the exercise-induced osteogenic effect appeared to be site specific $[29,30]$. The conflicting results in these studies, however, prevented a definite conclusion on the issue of the exerciseinduced osteogenic effect.

The purpose of this study was to investigate whether a 24-week program of a closely supervised aerobic highimpact loading exercise would be beneficial for physical fitness and BMD of L2-L4 and femoral neck in osteopenic postmenopausal women.

\section{Materials and Methods}

\section{Subjects}

Subjects were recruited through various advertising strategies, such as posters in physicians' offices and outpatient departments in 